\title{
ON THE CENTER CONDITIONS FOR ANALYTIC MONODROMIC DEGENERATE SINGULARITIES
}

\author{
JAUME GINÉ AND JAUME LLIBRE
}

\begin{abstract}
In this paper we present two methods for detecting centers of monodromic degenerate singularities of planar analytic vector fields. These methods use auxiliary symmetric vector fields can be applied independently that the singularity is algebraic solvable or not, or has characteristic directions or not. We remark that these are the first methods which allows to study monodromic degenerate singularities with characteristic directions.
\end{abstract}

\section{IntRoduCtion AND STATEMENT OF the MAIN RESUlts}

One of the classical problems in the qualitative theory of planar analytic differential systems in $\mathbb{R}^{2}$ is to characterize the local phase portrait near an isolated singular point. By using the blow-up technique the problem is solve except when the singularity is monodromic, i.e. the solutions of the differential equation in a neighborhood of the singularity turn around it either in forward time, or in backward time, see for instance $[9,11]$ and references therein.

When the differential system is analytic, a monodromic singular point is either a center or a focus, see [10, 21, 22]. A center is a singular point having a neighborhood filled of periodic orbits except by the singular point, and a focus is a singular point such that all the near orbits with the exception of the singular point spiral either in forward or in backward time to the singular point. The center problem consists on distinguishing between a center and a focus.

Let $p \in \mathbb{R}^{2}$ be a singular point of an analytic differential system in $\mathbb{R}^{2}$, and assume that $p$ is a center. Without loss of generality we can assume that $p$ is the origin of coordinates (if necessary we do a

1991 Mathematics Subject Classification. Primary 34C05. Secondary 58F14.

Key words and phrases. degenerate center, first integral, Bautin method, algebraically solvable.

The first author is partially supported by a MCYT/FEDER grant number MTM2008-00694 and by a CIRIT grant number 2009SGR 381. The second author is partially supported by a MCYT/FEDER grant number MTM2008-03437, by a CIRIT grant number 2009SGR 410 and by ICREA Academia. 
translation of coordinates sending $p$ to the origin). Then, after a linear change of variables and a rescaling of the time variable (if necessary), the system can be written in one of the following three forms:

$$
\begin{array}{lll}
\dot{x}=-y+F_{1}(x, y), & & \dot{y}=x+F_{2}(x, y) ; \\
\dot{x}=y+F_{1}(x, y), & & \dot{y}=F_{2}(x, y) ; \\
\dot{x}=F_{1}(x, y), & & \dot{y}=F_{2}(x, y)
\end{array}
$$

where $F_{1}(x, y)$ and $F_{2}(x, y)$ are real analytic functions without constant and linear terms, defined in a neighborhood of the origin. A center of an analytic differential system in $\mathbb{R}^{2}$ is called of the linear type, nilpotent or degenerate, if it can be written after an affine change of variables and a rescaling of time as system (1), (2) or (3), respectively.

The center problem for the linear type centers was already solved by Poincaré [32] and Lyapunov [23] in terms of the existence of an analytic first integral, see also [31]. The relation between the integrability problem and the center problem is studied in $[7,8]$.

For nilpotent singular points the monodromy problem was solved in [1], and the characterization of a nilpotent center in terms of its normal form is studied in $[5,30,33]$. In $[14,15]$ is proved that all the nilpotent centers are limit of linear type centers and consequently the Poincaré-Lyapunov method to find linear type centers can also be used to find all the nilpotent centers. Also in [14] it is showed that the degenerate centers which are limit of linear type centers are also detectable with the Poincaré-Lyapunov method, and that a Hamiltonian or time-reversible degenerate center is always limit of a linear type center. In [18] it is proved that a degenerate center (3) having an analytic inverse integrating factor $V(x, y)$ with $V(0,0) \neq 0$ is always limit of linear type centers.

In [25] it is shown that an isolated singular point $p$ of an analytic differential system is a center if and only if there exists a first integral of class $C^{\infty}$ with an isolated minimun at the singular point $p$. The next slightly different characterization of centers, given in [18], is a straightforward corollary of the previous characterization: a singular point $p$ of an analytic differential system is a center if and only if $p$ is monodromic and there exists a $C^{\infty}$ first integral defined in a neighborhood of $p$. In [19] is established that a degenerate analytic center is always limit of $\mathcal{C}^{\infty}$ linear type centers. If the degenerate center has an analytic first integral, then it is limit of linear type analytic centers. Unfortunately from this result is not possible to deduce an algorithm which provides the center conditions in function of the parameters of the system. 
The main difficulty in the problem of distinguishing between a center and a focus comes from the fact that this problem for degenerate centers may be algebraically non-solvable. We say that a center problem is algebraically non-solvable when does not exist an infinite sequence of independent polynomial expressions, involving the coefficients of the system, such that their simultaneous vanishing guarantees the existence of a center, whereas the non-nullity of any of these expressions implies non-existence of a center, see $[3,6]$. It was conjectured that the centers of analytic systems are analytically solvable, see [3], and recently it has been proved in [27]. Some partial results for the degenerate center problem can be found in $[12,13,16,17,24]$.

Let $n \geq 2$ be the minimum order of the lower terms of $F_{1}$ and $F_{2}$. We denote by $F_{1}^{n}$ and $F_{2}^{n}$ be the terms of order $n$ of $F_{1}$ and $F_{2}$ respectively, eventually one of these two polynomials can be zero. We say that $\theta$ is a characteristic direction of the singular point located at the origin of system (3) if

$$
\cos \theta F_{2}^{n}(\cos \theta, \sin \theta)-\sin \theta F_{1}^{n}(\cos \theta, \sin \theta)=0 .
$$

If there is an orbit of system (3) tending to the origin with a definite tangent at this point, then the direction defined by this tangent is a characteristic direction, and such an orbit is called a characteristic orbit. We recall that characteristic directions can or cannot have associated characteristic orbits. For more details see for instance $[2,11]$. Consequently a singular point of an analytic differential system is monodromic if it has no characteristic orbits, but it can have or not characteristic directions, see for more details [34]. There exist an algorithm that solves the stability problem for the monodromic degenerate singularities, see [28].

For the origin of system (3) without characteristic directions exists an algorithm for distinguishing between a center and a focus, called the Bautin method see [4] and also [16, 18], or the proof of Theorem 2 in section 3 where we use it. We recall that the Bautin method also determine non-algebraic center conditions, see for instance [16, 35]. Examples of non-algebraic solvable systems (3) when the singular point has no characteristic directions can be found in [20,35]. In [18] it is proved that system (3) with characteristic directions at the origin and when the lower order terms of system (3) are greater than 3 is limit of systems (3) without characteristic directions, and that the Bautin method can be applied to these last system for determining the center conditions of system (3). 
For systems (3) the center problem becomes more difficult when there exist characteristic directions. The main difficulty in this case is that the return map is no longer differentiable at the origin, see [3, 26, 29].

The aim of this work is to present two methods for studying the center problem for monodromic degenerate singularities algebraically solvable or not of planar analytic differential systems using auxiliary symmetric vector fields. In many cases these methods allow to decide if the singularity is a center. In particular these methods can be applied to monodromic degenerate singularities with characteristic directions. We remark that up to now for such singularities when the lower order terms of system (3) are equal 3 no method existed for studying the center problem.

Now we shall present the basic ideas of the methods for detecting centers that we will describe precisely later on. First, taking polar coordinates and an adequate rescaling of the independent variable we can write system (3) into the form

$$
\dot{r}=f(r, \theta), \quad \dot{\theta}=g(\theta)+r h(r, \theta) .
$$

It is well known that if $g(\theta)$ does not vanish we can apply the Bautin's algorithm for determine if the origin of system (3) is a center. Here we want to study the case when $g(\theta)$ vanishes.

Roughly speaking in the first method we consider the monodromic point $p$ at the origin of system (3) as a composition of two halves of time-reversible degenerate centers and for deciding if $p$ is a center or not we perturb the two time-reversible centers with linear terms of order $\varepsilon$. Thus the possible center at $p$ can be studied taking $\varepsilon \rightarrow 0$ the composition of a half of the Poincaré maps (computing the classical Poincaré-Liapunov constants) of the two perturbed time-reversible systems. In this case we only can detect the algebraic solvable centers.

The second method divides the monodromic point $p$ of system (3) as a composition of four time-reversible degenerate centers and for deciding if $p$ is a center or not we perturb the four time-reversible centers with lower order terms of order $\varepsilon$ in such away that the four perturbed systems have no characteristic directions. In this way the possible center at $p$ can be studied taking $\varepsilon \rightarrow 0$ the composition of a quarter of the Poincaré maps (using the Bautin's method) of the four perturbed time-reversible systems. In this case we can also detect the non-algebraic solvable centers.

Now we shall describe with detail both methods. The first method is based in Theorem 2, and the second one in Theorem 4 . 
It is known, see for instance [12] for a proof, that if an analytic differential system without linear terms has a focus or a center at the origin, then the degree of the lower terms is odd. Using this result it follows that an analytic differential system having a monodromic point at the origin can be written as

(4) $\dot{x}=P(x, y)=\sum_{i=n}^{\infty} P_{i}(x, y), \quad \dot{y}=Q(x, y)=\sum_{i=m}^{\infty} Q_{i}(x, y)$

with $s=\min \{n, m\} \geq 3$.

For the statement of Theorem 2 we need the following preliminary result.

Proposition 1. Consider the analytic differential system (4) with a monodromic degenerate singular point at the origin. We can always construct two analytic systems $S_{1}$ and $S_{2}$ both having a degenerate center at the origin, where $S_{1}$ is the pull-back of the half-plane $y>0$ through the map $y=z^{2}$, and $S_{2}$ is the pull-back of the half-plane $y<0$ through the map $y=-z^{2}$. By construction these two systems $S_{1}$ and $S_{2}$ are time-reversible systems and hence, are limit of linear type analytic centers.

Proposition 1 will be proved in section 2 .

Theorem 2. Consider the analytic differential systems (4) with a monodromic degenerate singular point $p$ at the origin. If the return map, obtained by composition of the return maps of the system $S_{1}$ in the half-plane $y>0$ and of the system $S_{2}$ in the half-plane $y<0$, is the identity $p$ is an algebraically solvable degenerate center.

Theorem 2 is proved in section 3 .

In the proof of Theorem 2 (see the end of section 3) we shall state the method for detecting algebraically solvable centers in a family of systems with a monodromic degenerate singular point. The conditions detected by the method will be algebraic, because we transform the initial center problem into a linear type center problem which always give algebraic conditions. So this method only can detect degenerate centers algebraically solvable. The method proposed is applied to one example in section 6, see Proposition 5.

Now the question that we shall consider is: how to develop a method for detecting the non-algebraic solvable degenerate centers with characteristic directions?

For the statement of Theorem 4 we need the next preliminary result. 
Proposition 3. Consider the analytic system (4) with a monodromic degenerate singular point at the origin. We construct four analytic systems $S_{1}, S_{2}, S_{3}$ and $S_{4}$ with a degenerate center at the origin associated to the first, second, third and four quadrant, respectively. We define $S_{1}$ as the pull-back of the half-plane $y>0$ through the map $y=z^{2}$ followed by the pull-back of the half-plane $x>0$ through the map $x=u^{2}$. We define $S_{2}$ as the pull-back of the half-plane $y>0$ through the map $y=z^{2}$ followed by the pull-back of the half-plane $x<0$ through the map $x=-u^{2}$. In a similar way we define systems $S_{3}$ and $S_{4}$. These four systems are time-reversible systems and hence, are limit of degenerate analytic centers without characteristic directions.

Now we can establish the following theorem which will allow to construct a method for studying any degenerated center. In particular this method allows to study the non-algebraic solvable monodromic degenerate singular point with characteristic directions.

Theorem 4. Consider a degenerate center with or without characteristic directions, algebraically solvable or not given by system (4) with a monodromic degenerate singular point $p$ at the origin. If the return map of the system, which is composition of the return maps of the system $S_{1}$ in the first quadrant, of the system $S_{2}$ in the second quadrant, of the system $S_{3}$ in the third quadrant and of the system $S_{4}$ in the fourth quadrant, is the identity $p$ is a degenerate center.

Proposition 3 and Theorem 4 are proved in Section 4 and 6 respectively.

From Theorem 4 follows a method for finding center conditions for every monodromic degenerate singular point. Of course the computations associated to the methods can be very tedious, and sometimes impossible to end. This already occurs with the computations of the standard Poincaré-Liapunov constants for determining the linear type centers or for the Bautin method for determining the degenerate centers without characteristic directions, the first difficulty is to compute the constants, and the second is to obtain from them the center conditions. In Section 6 we apply the method based in Theorem 4 to an example, see Proposition 6.

\section{Proof of Proposition 1}

Consider system (4) with a monodromic degenerate singular point at the origin. Assume that the dot in system (4) denotes derivative with respect to the time $t$. First in the half-plane $y>0$ we change the variables from $(x, y, t)$ to $(x, z, s)$ through $y=z^{2}$ and $d t=2 z d s$, where 
$s$ will be the new independent variable (or time) of the differential system. In the new variables system (4) in $y>0$ becomes the system $S_{1}$ equal to

$$
\dot{x}=2 z P\left(x, z^{2}\right), \quad \dot{z}=Q\left(x, z^{2}\right),
$$

in $z>0$. System $S_{1}$ is a time-reversible system invariant by the reversibility $(x, z, t) \rightarrow(x,-z,-t)$.

Second in the half-plane $y<0$ we change the variables from $(x, y, t)$ to $(x, z, s)$ through $y=-z^{2}$ and $d t=-2 z d s$. In the new variables system (4) in $y<0$ becomes the system $S_{2}$ equal to

$$
\dot{x}=-2 z P\left(x,-z^{2}\right), \quad \dot{z}=Q\left(x,-z^{2}\right),
$$

in $z<0$. System $S_{2}$ is also a time-reversible system invariant by the reversibility $(x, z, t) \rightarrow(x,-z,-t)$. Now applying the results proved in [14] (and mentioned in the introduction) we have that systems $S_{1}$ and $S_{2}$ are limit of linear type centers of the form

$$
\dot{x}=-\varepsilon z+2 z P\left(x, z^{2}\right), \quad \dot{z}=\varepsilon x+Q\left(x, z^{2}\right),
$$

and

$$
\dot{x}=-\varepsilon z-2 z P\left(x,-z^{2}\right), \quad \dot{z}=\varepsilon x+Q\left(x,-z^{2}\right),
$$

respectively. Here $\varepsilon$ needs to be small and positive. This completes the proof of Proposition 1.

\section{Proof of Theorem 2}

Consider system (4) with a monodromic degenerate singular point at the origin. Doing the process described in the proof of Proposition 1 , we arrive to system (5) and (6). Due to the reversibility the singular points located at the origin of these systems when they are considered in the whole plane $(x, z)$ are centers.

A standard method for distinguishing between a focus and a center is based on the computation of the derivatives of the return map at the origin. These derivatives are obtained by solving a system of recursive differential equations. This technique, called the Bautin method, will be used in what follows for studying the center problem at the origin of system (4), so we quickly recall it. Take polar coordinates $(r, \theta)$, defined by $x=r \cos \theta, y=r \sin \theta$, in system (5) or (6). In these coordinates both systems can be written as

$$
\frac{d r}{d \theta}=f(r, \theta)=f_{2}(\theta) r^{2}+f_{3}(\theta) r^{3}+\cdots
$$


where $f$ is analytic and for all $k \geq 2$, the functions $f_{k}$ are trigonometrical polynomials. Let

$$
r=r\left(\theta, r_{0}\right):=r_{0}+u_{2}(\theta) r_{0}^{2}+u_{3}(\theta) r_{0}^{3}+\cdots,
$$

be the solution of system (7) satisfying $r\left(0, r_{0}\right)=r_{0}$. Taking $\theta=0$ we obtain the initial conditions $u_{k}(0)=0$ for all $k \geq 2$. Substituting expression (8) in (7) we get a sequence of recursive differential systems $d u_{k} / d \theta$, for $k=2,3, \ldots$, which will allow to compute the functions $u_{k}(\theta)$, and consequently the return map of system (7) is given by

$$
\begin{aligned}
r\left(2 \pi, r_{0}\right) & =r_{0}+u_{2}(2 \pi) r_{0}^{2}+u_{3}(2 \pi) r_{0}^{3}+\cdots \\
& :=r_{0}+v_{2} r_{0}^{2}+v_{3} r_{0}^{3}+\cdots
\end{aligned}
$$

The values $v_{k}$, for $k \geq 2$, are called the focal values of the singular point located at the origin.

It is well known that either all the values $v_{k}$ vanish for $k \geq 2$, and in this case the origin is a center, or otherwise the first non zero focal value corresponds to some $k=2 m+1$ odd, and in this case the origin is a focus. In this situation it is said that the origin is a weak focus of order $m$ and the value $v_{2 m+1}$ is called the $m$-th Lyapunov constant of the system. Notice that the sign of this Lyapunov constant gives the stability of the origin. In fact if we do these computations for systems (5) or (6) we will obtain that all the values $v_{k}$ are null because both systems have a center at the origin due to their reversibility.

Our objective is to study when the singular point located at the origin of system (4) is a center or not. For doing that we shall work with the return map of system (4) around its origin. Due to the construction of systems of $S_{1}$ and $S_{2}$ this return map is the composition of the following two return maps. The return map of system $S_{1}$ from the positive $x$-axis to the negative $x$-axis in the half-plane $z>0$, with the return map of system $S_{2}$ from the negative $x$-axis to the positive $x$-axis in the half-plane $z<0$. But since systems $S_{1}$ and $S_{2}$ are limit when $\varepsilon \rightarrow 0$ of systems (5) and (6) respectively, we can study the return map of system (4) as the limit when $\varepsilon \rightarrow 0$ of the composition of the return maps of the system (5) in polar coordinates from $\theta=0$ to $\theta=\pi$, with the return map of the system (6) in polar coordinates from $\theta=\pi$ to $\theta=2 \pi$.

Evaluating the solution of system (5) at $\pi$ we obtain

$$
\begin{aligned}
r\left(\pi, r_{0}\right) & =r_{0}+u_{k}(\pi) r_{0}^{k}+\cdots \\
& :=r_{0}+w_{k} r_{0}^{k}+\cdots \\
& :=\widetilde{r}_{0}
\end{aligned}
$$


where $k=2 m+1$ correspond to the first non zero $w_{i}$. After we evaluate the solution of (6) with the initial condition $\theta=\pi$ and $r=\widetilde{r}_{0}$ at $\theta=2 \pi$. The result is the return Poincaré map depending on $\varepsilon$ whose limit when $\varepsilon \rightarrow 0$ will provide the return map of system (4) around the origin.

To study the solution of (6) with the initial condition $\theta=\pi$ and $r=\widetilde{r}_{0}$ at $\theta=2 \pi$, we take the polar coordinates $(r, \theta)$ defined by $x=r \cos (\theta+\pi), y=r \sin (\theta+\pi)$ in system (6), and we evaluate the solution of the new system with the initial condition $\theta=0$ and $r=\widetilde{r}_{0}$ at $\theta=\pi$. Thus we obtain

$$
\begin{aligned}
r\left(\pi, \widetilde{r}_{0}\right) & =\widetilde{r}_{0}+\bar{u}_{\ell}(\pi) \widetilde{r}_{0}^{\ell}+\cdots \\
& :=\widetilde{r}_{0}+\bar{w}_{\ell} \widetilde{r}_{0}^{\ell}+\cdots
\end{aligned}
$$

where $\ell=2 n+1$ correspond to the first non zero $\bar{w}_{i}$. Substituting the value of $\widetilde{r}_{0}$ we obtain

$$
\begin{aligned}
r\left(\pi, \widetilde{r}_{0}\right) & =\widetilde{r}_{0}+\bar{w}_{\ell} \widetilde{r}_{0}^{\ell}+\cdots \\
& =r_{0}+w_{k} r_{0}^{k}+\bar{w}_{l}\left(r_{0}+w_{k} r_{0}^{k}+\cdots\right)^{\ell}+\cdots \\
& =r_{0}+w_{k} r_{0}^{k}+\bar{w}_{l} r_{0}^{l}+\cdots
\end{aligned}
$$

Hence if the limit of $r_{0}+w_{k} r_{0}^{k}+\bar{w}_{l} r_{0}^{l}+\cdots$ when $\varepsilon \rightarrow 0$ is different from $r_{0}$, the origin of system (4) is a focus, otherwise it is a center, and the proof of Theorem 2 is done. In short we have a method to detect algebraically solvable degenerate centers.

\section{Proof of Proposition 3}

Consider system (4) with a monodromic degenerate singular point at the origin and with $s \geq 3$, this last condition is necessary in order to have a monodromic degenerate singular point as we have mentioned in section 1. First in the first quadrant $x>0$ and $y>0$ we change the variables from $(x, y, t)$ to $(x, z, s)$ through $y=z^{2}$ and $d t=2 z d s$. In the new variables system (4) in $x>0$ and $y>0$ becomes

$$
\dot{x}=2 z P\left(x, z^{2}\right), \quad \dot{z}=Q\left(x, z^{2}\right),
$$

in $x>0$ and $z>0$. To this last system in $x>0$ and $z>0$ we change the variables from $(x, z, s)$ to $(u, z, T)$ through $x=u^{2}$ and $d s=2 u d T$. In the new variables the last system goes over system $S_{1}$ equal to

$$
\dot{u}=2 z P\left(u^{2}, z^{2}\right), \quad \dot{z}=2 u Q\left(u^{2}, z^{2}\right) .
$$

System $S_{1}$ is a time-reversible system invariant by two reversibilities: $(u, z, t) \rightarrow(u,-z,-t)$ and $(u, z, t) \rightarrow(-u, z,-t)$. In the second quadrant $x<0$ and $y>0$ we change the variables from $(x, y, t)$ to $(x, z, s)$ 
through $y=z^{2}$ and $d t=2 z d s$. In the new variables system (4) in $x<0$ and $y>0$ becomes

$$
\dot{x}=2 z P\left(x, z^{2}\right), \quad \dot{z}=Q\left(x, z^{2}\right),
$$

in $x<0$ and $z>0$. To this last system in $x<0$ and $z>0$ we change the variables from $(x, z, s)$ to $(u, z, T)$ through $x=-u^{2}$ and $d s=-2 u d T$. In the new variables the last system goes over system $S_{2}$ equal to

$$
\dot{u}=2 z P\left(-u^{2}, z^{2}\right), \quad \dot{z}=-2 u Q\left(-u^{2}, z^{2}\right) .
$$

System $S_{2}$ is also a time-reversible system invariant by two reversibilities: $(u, z, t) \rightarrow(u,-z,-t)$ and $(u, z, t) \rightarrow(-u, z,-t)$.

In a similar way we construct systems $S_{3}$ and $S_{4}$ in the other quadrants. Moreover all systems $S_{1}, S_{2}, S_{3}$ and $S_{4}$ began with terms of at least fourth order. Hence applying the results proved in $[14,18]$ (and mentioned in the first section), we have that these four systems are limit of degenerate centers without characteristic directions of the form

$$
\begin{array}{cc}
\dot{u}=-\varepsilon z^{3}+2 z P\left(u^{2}, z^{2}\right), & \dot{z}=\varepsilon u^{3}+2 u Q\left(u^{2}, z^{2}\right) ; \\
\dot{u}=-\varepsilon z^{3}+2 z P\left(-u^{2}, z^{2}\right), & \dot{z}=\varepsilon u^{3}-2 u Q\left(-u^{2}, z^{2}\right) ; \\
\dot{u}=-\varepsilon z^{3}-2 z P\left(-u^{2},-z^{2}\right), & \dot{z}=\varepsilon u^{3}-2 u Q\left(-u^{2},-z^{2}\right) ; \\
\dot{u}=-\varepsilon z^{3}-2 z P\left(u^{2},-z^{2}\right), & \dot{z}=\varepsilon u^{3}+2 u Q\left(u^{2},-z^{2}\right) ;
\end{array}
$$

respectively, where $\varepsilon$ needs to be small and positive. So Proposition 3 is proved.

\section{Proof of TheOrem 4}

The proof of Theorem 4 follows in a similar way to the proof of Theorem 2.

Consider system (4) with a monodromic degenerate singular point at the origin. Doing the process described in the proof of Proposition 3 we arrive to systems (9), (10), (11) and (12), in the first, second, third and fourth quadrant respectively. Do to the reversibility of these systems when they are considered in the whole plane $(u, z)$ their origin are centers.

Again our objective is to study when the singular point located at the origin of system (4) is a center or not. For doing that we shall work with the return map of system (4) around the origin. Due to the construction of systems of $S_{1}, S_{2}, S_{3}$ and $S_{4}$ this return map is the composition of the following four return maps. The return map of 
system $S_{1}$ from the positive $u$-axis to the positive $z$-axis in the first quadrant, with the return map of system $S_{2}$ from the positive $z$-axis to the negative $u$-axis in the second quadrat, with the return map of system $S_{3}$ from the negative $u$-axis to the negative $z$-axis in the third quadrat, and finally with the return map of system $S_{4}$ from the negative $z$-axis to the positive $u$-axis in the fourth quadrant. But since systems $S_{1}, S_{2}, S_{3}$ and $S_{4}$ are limit when $\varepsilon \rightarrow 0$ of four systems (9), (10), (11) and (12) without characteristic directions at the origin, we can study the return map of system (4) as the limit when $\varepsilon \rightarrow 0$ of the composition of the return maps of those four systems.

The Bautin method also works for studying the return map of every one of the four systems (9), (10), (11) and (12), because these systems have no characteristic directions. Hence taking polar coordinates as in the proof of Theorem 2, all systems (9), (10), (11) and (12) take the form

$$
\frac{d r}{d \theta}=f(r, \theta)=f_{1}(\theta) r+f_{2}(\theta) r^{2}+\cdots
$$

where $f$ is analytic and for all $k \geq 1$, the functions $f_{k}$ are quotients of trigonometrical polynomials. As before, we propose a solution of (13) of the form

$$
r=r\left(\theta, r_{0}\right):=r_{0}+u_{2}(\theta) r_{0}^{2}+u_{3}(\theta) r_{0}^{3}+\cdots,
$$

satisfying $r\left(0, r_{0}\right)=r_{0}$. Taking $\theta=0$ we obtain the initial conditions $u_{k}(0)=0$ for all $k \geq 2$. Substituting expression (14) in (13) we get a sequence of recursive differential system for computing $u_{k}(\theta)$ and the return map for one of the systems (9), (10), (11) and (12) is given by

$$
\begin{aligned}
r\left(2 \pi, r_{0}\right) & =r_{0}+u_{2}(2 \pi) r_{0}^{2}+u_{3}(2 \pi) r_{0}^{3}+\cdots \\
& :=r_{0}+v_{2} r_{0}^{2}+v_{3} r_{0}^{3}+\cdots
\end{aligned}
$$

where the values $v_{k}, k \geq 2$, are called the generalized focal values of the origin. In fact, if we do these computations for systems (9), (10), (11) and (12) we will obtain that all the values $v_{k}$ are null because systems (9), (10), (11) and (12) have a center at the origin due to their reversibility.

Our objective is to study when the singular point located at the origin of system (4) is a center or not. For doing that we shall work with the return map of system (4) around the origin. Since systems $S_{1}, S_{2}, S_{3}$ and $S_{4}$ are limit when $\varepsilon \rightarrow 0$ of systems (9), (10), (11) and (12) respectively, we can study the return map of system (4) as the limit when $\varepsilon \rightarrow 0$ of the composition of the return maps of the system (9) in polar coordinates from $\theta=0$ to $\theta=\pi / 2$, with the return map of the system (10) in polar coordinates from $\theta=\pi / 2$ to $\theta=\pi$, with 
the return map of the system (11) in polar coordinates from $\theta=\pi$ to $\theta=3 \pi / 3$, and with the return map of the system (12) in polar coordinates from $\theta=3 \pi / 2$ to $\theta=2 \pi$.

Doing the composition of the return maps of the four systems (9), (10), (11) and (12) to implement the same process described in Section 3. We first use system (9) evaluating its solution at $\pi / 2$ we obtain

$$
\begin{aligned}
r\left(\pi / 2, r_{0}\right) & =r_{0}+u_{k}(\pi / 2) r_{0}^{k}+\cdots \\
& :=r_{0}+w_{k} r_{0}^{k}+\cdots, \\
& :=\widetilde{r}_{0},
\end{aligned}
$$

where $k=2 m+1$ correspond to the first non zero $w_{i}$. Now, we have that the values $w_{i}$ can be different from zero for $k \geq 2$. Second we evaluate the solution of next system (10) at the initial condition $\widetilde{r}_{0}$, from $\pi / 2$ to $\pi$. Repeating this process to make a complete turn with the corresponding system at each quadrant and doing the compositions of the four return maps in the same way that in the proof of Theorem 2 we obtain in general a non-algebraic condition in order to have a center for the initial system (4). This condition can be non-algebraic because the Bautin method, in general, gives non-algebraic conditions, see for instance [18]. In short if the return map of the composition of the four return maps is the identity we have a center, and the proof of Theorem 4 is done. Thus we have a method for detecting when a monodromic degenerate singular point is a center, algebraically solvable or not, with or without characteristic directions.

\section{Applications}

We now analyze some examples in order to show how works the methods that follow from Theorems 2 and 4 . It is important to remark that the methods described in this paper only can be applied to monodromic singular points.

We consider the differential system

$$
\begin{aligned}
& \dot{x}=y\left(a x^{2}+b x y+c y^{2}\right), \\
& \dot{y}=y^{2}(a x+b y)+x^{5} .
\end{aligned}
$$

with $a \leq 0$ and $c<0$. Using the theory presented in [12], the origin is a monodromic singular point if $a<0$ and $c<0$. The monodromy of system (15) is also studied in [11], where it is proved that if $a=0$ and $b \neq 0$ then the origin is not monodromic. In [19] it is studied when the origin of system (15) has a center with an analytic first integral. Using the method derived from section 3 we have for system (15) the next result. 
Proposition 5. System (15) has a center at the origin if and only if $a=b=0$ and $c d<0$.

Proof. System (15) is a degenerate system with the characteristic direction $y=0$, because $x Q_{3}-y P_{3}=-c y^{4}$ being $P_{3}$ and $Q_{3}$ the homogeneous parts of degree 3 of system (15). First we construct systems (5) and (6) for system (15) and we obtain

(16) $\dot{x}=-\varepsilon z+2 z^{3}\left(a x^{2}+b x z^{2}+c z^{4}\right), \quad \dot{z}=\varepsilon x+d x^{5}+z^{4}\left(a x+b z^{2}\right)$,

and

$$
\dot{x}=-\varepsilon z+2 z^{3}\left(a x^{2}-b x z^{2}+c z^{4}\right), \quad \dot{z}=\varepsilon x+d x^{5}+z^{4}\left(a x-b z^{2}\right),
$$

respectively. If we apply the method described in section 3 and construct the return map for system (16) we obtain

$$
r\left(\pi, r_{0}\right)=r_{0}+\frac{128 b}{105 \varepsilon} r_{0}^{6}+\cdots=\widetilde{r}_{0} .
$$

After we evaluate the solution of (17) with the initial condition $\theta=\pi$ and $r=\widetilde{r}_{0}$ at $\theta=2 \pi$. To compute that, we take the polar coordinates $(r, \theta)$ defined by $x=r \cos (\theta+\pi), y=r \sin (\theta+\pi)$ in system (17), and we evaluate the solution of the new system with the initial condition $\theta=0$ and $r=\widetilde{r}_{0}$ at $\theta=\pi$. Thus we obtain

$$
\begin{aligned}
r\left(\pi, \widetilde{r}_{0}\right) & =\widetilde{r}_{0}+\frac{128 b}{105 \varepsilon} \widetilde{r}_{0}^{6}+\cdots \\
& =r_{0}+\frac{128 b}{105 \varepsilon} r_{0}^{6}+\frac{128 b}{105 \varepsilon}\left(r_{0}+\frac{128 b}{105 \varepsilon} r_{0}^{6}+\cdots\right)^{6}+\cdots \\
& =r_{0}+\frac{256 b}{105 \varepsilon} r_{0}^{6}+\cdots
\end{aligned}
$$

Consequently a necessary condition to have a center is $b=0$. If we impose $b=0$ we do not find any more conditions. This is because the original system (15) now takes the form

$$
\dot{x}=y\left(a x^{2}+c y^{2}\right), \quad \dot{y}=a x y^{2}+x^{5} .
$$

System (18) is a time-reversible system invariant by two reversibilities: $(x, y, t) \rightarrow(-x, y,-t)$ and $(x, y, t) \rightarrow(x,-y,-t)$. Hence this system is symmetric respect to the $x$ - and the $y$-axis. Therefore we must apply the method described in section 3 but, for instance, with respect to the first-quadrant bisector. First, in the half-plane $y-x>0$ we make the change the variables from $(x, y, t)$ to $(x, z, s)$ through $y-x=z^{2}$ and $d t=2 z d s$. In the new variables system (18) in $y-x>0$ becomes

$$
\begin{aligned}
& \dot{x}=2 z\left(x+z^{2}\right)\left(a x^{2}+c\left(x+z^{2}\right)^{2}\right), \\
& \dot{z}=d x^{5}+a x\left(x+z^{2}\right)^{2}-\left(x+z^{2}\right)\left(a x^{2}+c\left(x+z^{2}\right)^{2}\right),
\end{aligned}
$$


in $z>0$. Second, in the half-plane $y-x<0$ we change the variables from $(x, y, t)$ to $(x, z, s)$ through $y-x=-z^{2}$ and $d t=-2 z d s$. In the new variables system (18) in $y-x<0$ becomes

$$
\begin{aligned}
& \dot{x}=-2 z\left(x-z^{2}\right)\left(a x^{2}+c\left(x-z^{2}\right)^{2}\right), \\
& \dot{z}=d x^{5}+a x\left(x-z^{2}\right)^{2}-\left(x-z^{2}\right)\left(a x^{2}+c\left(x-z^{2}\right)^{2}\right),
\end{aligned}
$$

in $z<0$.

Now we apply the method described in section 3 and construct the return map for system (19) obtaining

$$
r\left(\pi, r_{0}\right)=r_{0}+\frac{16 a}{15 \varepsilon} r_{0}^{4}+\frac{32 a c}{105 \varepsilon^{2}} r_{0}^{6}+\cdots=\widetilde{r}_{0} .
$$

After we evaluate the solution of (20) with the initial conditions $\theta=\pi$ and $r=\widetilde{r}_{0}$ at $\theta=2 \pi$. To compute that, as before, we take the polar coordinates $(r, \theta)$ defined by $x=r \cos (\theta+\pi), y=r \sin (\theta+\pi)$ in system (17), and we evaluate the solution of the new system with the initial conditions $\theta=0$ and $r=\widetilde{r}_{0}$ at $\theta=\pi$. Thus we obtain

$$
r\left(\pi, \widetilde{r}_{0}\right)=\widetilde{r}_{0}+\frac{16 a}{15 \varepsilon} \widetilde{r}_{0}^{4}+\frac{32 a c}{105 \varepsilon^{2}} \widetilde{r}_{0}^{6}+\cdots
$$

We substitute the value of $\widetilde{r}_{0}$ and the composition has the form

$$
\begin{aligned}
r\left(\pi, \widetilde{r}_{0}\right) & =\widetilde{r}_{0}+\frac{16 a}{15 \varepsilon} \widetilde{r}_{0}^{4}+\cdots \\
& =r_{0}+\frac{16 a}{15 \varepsilon} r_{0}^{4}+\frac{16 a}{15 \varepsilon}\left(r_{0}+\frac{16 a}{15 \varepsilon} r_{0}^{4}+\cdots\right)^{4}+\cdots \\
& =r_{0}+\frac{32 a}{15 \varepsilon} r_{0}^{4}+\cdots
\end{aligned}
$$

Therefore we obtain that the second necessary condition is $a=0$. If $a=b=0$ system (15) is Hamiltonian with the first integral $H(x, y)=$ $2 d x^{6}-3 c y^{4}$. System (15) with $a=b=0$ has a degenerate center with characteristic directions at the origin if $c d<0$ because in this case the origin is surrounded by ovals.

Now we consider the differential system

$$
\begin{aligned}
& \dot{x}=b x^{2} y+a x y^{2}-b y^{3}-x^{4} \\
& \dot{y}=4 b x y^{2}-a y^{3}+2 x^{5} .
\end{aligned}
$$

System (21) is a degenerate system with the characteristic direction $y=0$, because $x Q_{3}-y P_{3}=b y^{2}\left(3 x^{2}+y^{2}\right)$ where $P_{3}$ and $Q_{3}$ are the lower homogeneous parts of system (21). Hence order of the lower terms is $s=3$, and we cannot apply the method developed in [18]. By using the blow-up technique in [11] it was shown that the origin of system (21) is monodromic if and only if $b \geq 1 / 4$. Now we shall 
see that using the method described in section 6 we can establish the following result.

Proposition 6. System (21) has never a center at the origin.

Proof. First we construct systems (9), (10), (11) and (12) for system (21), and we obtain

$$
\begin{aligned}
& \dot{u}=-\varepsilon z^{3}+2 z\left(-u^{8}+b u^{4} z^{2}+a u^{2} z^{4}-b z^{6}\right), \\
& \dot{z}=\varepsilon x^{3}+2 u\left(2 u^{10}+4 b u^{2} z^{4}-a z^{6}\right), \\
& \dot{u}=-\varepsilon z^{3}+2 z\left(-u^{8}+b u^{4} z^{2}-a u^{2} z^{4}-b z^{6}\right), \\
& \dot{z}=\varepsilon x^{3}-2 u\left(-2 u^{10}-4 b u^{2} z^{4}-a z^{6}\right), \\
& \dot{u}=-\varepsilon z^{3}-2 z\left(-u^{8}-b u^{4} z^{2}-a u^{2} z^{4}+b z^{6}\right), \\
& \dot{z}=\varepsilon x^{3}-2 u\left(-2 u^{10}-4 b u^{2} z^{4}+a z^{6}\right),
\end{aligned}
$$

and

$$
\begin{aligned}
& \dot{u}=-\varepsilon z^{3}-2 z\left(-u^{8}-b u^{4} z^{2}+a u^{2} z^{4}+b z^{6}\right), \\
& \dot{z}=\varepsilon x^{3}+2 u\left(2 u^{10}+4 b u^{2} z^{4}+a z^{6}\right),
\end{aligned}
$$

respectively. If we apply the method described in section 6 and construct the return map from $\theta=0$ to $\theta=\pi / 2$ for system (22) we obtain

$$
r\left(\pi / 2, r_{0}\right)=r_{0}+\frac{8 b-a \pi}{8 \varepsilon} r_{0}^{5}-\frac{8}{15 \varepsilon} r_{0}^{7}+\cdots=\widetilde{r}_{0} .
$$

After we evaluate the solution of (23) with the initial conditions $\theta=$ $\pi / 2$ and $r=\widetilde{r}_{0}$ at $\theta=\pi$. To compute that, we take the polar coordinates $(r, \theta)$ defined by $x=r \cos (\theta+\pi / 2), y=r \sin (\theta+\pi / 2)$ in system (23), and we evaluate the solution of the new system with the initial conditions $\theta=0$ and $r=\widetilde{r}_{0}$ at $\theta=\pi / 2$. Thus we obtain

$$
r\left(\pi, \widetilde{r}_{0}\right)=\widetilde{r}_{0}-\frac{8 b+a \pi}{8 \varepsilon} \widetilde{r}_{0}^{5}+\frac{8}{15 \varepsilon} \widetilde{r}_{0}^{7}+\cdots=\widetilde{\widetilde{r}}_{0} .
$$

Next step is to evaluate the solution of (24) with the initial conditions $\theta=\pi$ and $r=\widetilde{\widetilde{r}}_{0}$ at $\theta=3 \pi / 2$. To compute that, we take the polar coordinates $(r, \theta)$ defined by $x=r \cos (\theta+\pi), y=r \sin (\theta+\pi)$ in system (24), and we evaluate the solution of the new system with the initial conditions $\theta=0$ and $r=\widetilde{\widetilde{r}}_{0}$ at $\theta=\pi / 2$. Thus we obtain

$$
r\left(3 \pi / 2, \widetilde{\widetilde{r}}_{0}\right)=\widetilde{\widetilde{r}}_{0}+\frac{8 b-a \pi}{8 \varepsilon} \widetilde{\widetilde{r}}_{0}^{5}+\frac{8}{15 \varepsilon} \widetilde{\widetilde{r}}_{0}^{7}+\cdots=\widetilde{\widetilde{r}}_{0} .
$$

Finally we evaluate the solution of (25) with the initial conditions $\theta=$ $3 \pi / 2$ and $r=\widetilde{\widetilde{r}}_{0}$ at $\theta=2 \pi$. To compute that, we take the polar coordinates $(r, \theta)$ defined by $x=r \cos (\theta+3 \pi / 2), y=r \sin (\theta+3 \pi / 2)$ 
in system (25), and we evaluate the solution of the new system with the initial conditions $\theta=0$ and $r=\widetilde{\widetilde{r}}_{0}$ at $\theta=\pi / 2$. Thus we obtain

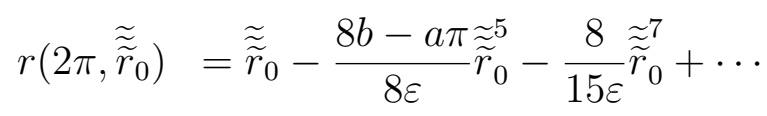

The composition of these four return maps gives the condition $a=0$. The terms in $r_{0}^{7}$ cancel and if we repeat the computations of the four returns maps with $a=0$ we have

$$
\begin{aligned}
& r\left(\pi / 2, r_{0}\right)=r_{0}+\frac{b}{\varepsilon} r_{0}^{5}-\frac{8}{15 \varepsilon} r_{0}^{7}+\frac{b^{2}+\varepsilon}{3 e^{2}} r_{0}^{9}+\cdots=\widetilde{r}_{0}, \\
& r\left(\pi, \widetilde{r}_{0}\right)=\widetilde{r}_{0}-\frac{b}{\varepsilon} \widetilde{r}_{0}^{5}+\frac{8}{15 \varepsilon} \widetilde{r}_{0}^{7}+\frac{13 b^{2}-\varepsilon}{3 e^{2}} \widetilde{r}_{0}^{9}+\cdots=\widetilde{\widetilde{r}}_{0}, \\
& r\left(3 \pi / 2, \widetilde{\widetilde{r}}_{0}\right)=\widetilde{\widetilde{r}}_{0}+\frac{b}{\varepsilon} \widetilde{r}_{0}^{5}+\frac{8}{15 \varepsilon} \widetilde{\widetilde{r}}_{0}^{7}+\frac{b^{2}+\varepsilon}{3 e^{2}} \widetilde{r}_{0}^{9}+\cdots=\widetilde{\widetilde{r}}_{0}, \\
& r\left(2 \pi, \widetilde{\widetilde{r}}_{0}\right)=\widetilde{\widetilde{r}}_{0}-\frac{b \approx_{\tilde{r}}^{5}}{\varepsilon}-\frac{8}{15 \varepsilon} \approx_{\widetilde{r}_{0}^{7}}^{7}+\frac{13 b^{2}-\varepsilon}{3 e^{2}} \approx_{\widetilde{r}_{0}^{9}}^{9}+\cdots
\end{aligned}
$$

Therefore, after the composition of these four maps, the second necessary condition is $b=0$ in contradiction with the monodromy condition $b \geq 1 / 4$. In fact, for $a=b=0$ the system has a straight line $x=0$ passing through the origin.

\section{REFERENCES}

[1] A. F. AndREev, Solution of the problem of the center and the focus in one case. (Russian) Akad. Nauk SSSR. Prikl. Mat. Meh. 17, (1953). 333-338.

[2] A.A. Andronov, E.A. Leontovich, I.I. Gordon \& A.G. Maier, Qualitative theory of second-order dynamic systems. John Wiley \& Sons, New YorkToronto, Israel Program for Scientific Translations, Jerusalem-London, 1973.

[3] D.V. Anosov, S.Kh. Aranson, V.I.Arnold, I.U. Bronshtein, V.Z. Grines \& YU. S. IL'Yashenko, Ordinary differential equations and smooth dynamical systems, Springer-Verlag, Berlin, 1997.

[4] N.N. BAUtin, On the number of limit cycles which appear with the variation of coefficients from an equilibrium position of focus or center type, Mat. Sb. 30 (72) (1952), 181-196; Amer. Math. Soc. Transl. 100 (1954) 397-413.

[5] M. Berthier \& R. Moussu, Réversibilité et classification des centres nilpotents, Ann. Inst. Fourier (Grenoble) 44 (1994), 465-494.

[6] A.D. Bruno, Local methods in nonlinear differential equations, Springer Series in Soviet Mathematics. Springer-Verlag, Berlin, 1989.

[7] J. Chavarriga, H. Giacomini, J. Giné \& J. Llibre, On the integrability of two-dimensional flows, J. Differential Equations 157 (1999), 163-182.

[8] J. Chavarriga, H. Giacomini, J. Giné \& J. Llibre, Local analytic integrability for nilpotent centers, Ergodic Theory Dynam. Systems 23 (2003), 417-428.

[9] F. Dumortier, J. Llibre \& J.C. Artés, Qualitative theory of planar differential systems, UniversiText, Springer-Verlag, New York, 2006. 
[10] J. ÉCALLE, Introduction aux fonctions analysables et preuve constructive de la conjecture de Dulac. Actualités Mathématiques. Hermann, Paris, 1992.

[11] I.A. García, J. Giné \& M. Grau, A necessary condition in the monodromy problem for analytic differential equations on the plane, J. Symbolic Comput. 41 (2006), no. 9, 943-958.

[12] A. Gasull, J. Llibre, V. Mañosa \& F. Mañosas, The focus-center problem for a type of degenerate systems, Nonlinearity 13 (2000), 699-730.

[13] A. Gasull, V. Mañosa \& F. MAÑosas, Monodromy and stability of a generic class of degenerate planar critical points, J. Differential Equations $\mathbf{1 8 2}$ (2002), no. 1, 169-190.

[14] H. Giacomini, J. Giné \& J. LliBre, The problem of distinguishing between a center an a focus for nilpotent and degenerate analytic systems, J. Differential Equations 227 (2006), no. 2, 406-426.

[15] H. Giacomini, J. Giné \& J. Llibre, Corrigendum to: "The problem of distinguishing between a center and a focus for nilpotent and degenerate analytic systems", J. Differential Equations 232 (2007), no. 2, 702-702.

[16] J. Giné, Sufficient conditions for a center at completely degenerate critical point, Internat. J. Bifur. Chaos Appl. Sci. Engrg. 12 (2002), 1659-1666.

[17] J. Giné, Analytic integrability and characterization of centers for generalized nilpotent singular points, Appl. Math. Comput. 148 (2004), no. 3, 849-868.

[18] J. Giné, On the centers of planar analytic differential systems, Internat. J. Bifur. Chaos Appl. Sci. Engrg. 17 (2007), 3061-3070.

[19] J. Giné, On the degenerate center problem, preprint, Universitat de Lleida, 2010.

[20] YU.S. IL'YASHENKO, Algebraic nonsolvability and almost algebraic solvability of the center-focus problem, Functional Anal. Appl. 6 (1972), no. 3, 30-37.

[21] Yu.S. IL'YAshenko, Finiteness theorems for limit cycles. Translated from the Russian by H. H. McFaden. Translations of Mathematical Monographs, 94. American Mathematical Society, Providence, RI, 1991.

[22] Y. IlyASHENKo \& S. YAKOVENKO, Lectures on analytic differential equations, Graduate Studies in Mathematics 86, Amer. Math. Soc., Providence, RI, 2008.

[23] M.A. Lyapunov Problème général de la stabilité du mouvement, Ann. of Math. Stud. 17, Princeton University Press, 1947.

[24] V. MAÑosa On the center problem for degenerate singular points of planar vector fields, Internat. J. Bifur. Chaos Appl. Sci. Engrg. 12 (2002), no. 4, 687-707.

[25] L. Mazzi \& M. Sabatini, A characterization of centres via first integrals, J. Differential Equations 76 (1988), 222-237.

[26] N.B. Medvedeva, The principal term of the monodromy transformation of a monodromic singular point is linear, Siberian Math. J. 33 (1992), 280-288

[27] N.B. Medvedeva, On the analytic solvability of the problem of distinguishing between a center and a focus, (Russian) Dokl. Akad. Nauk 394 (2004), no. 6, 735-738.

[28] N.B. Medvedeva, On the analytic solvability of the problem of distinguishing between a center and a focus, Proc. Steklov Inst. Math. 254 (2006), no. 3, 7-93. 
[29] N.B. Medvedeva \& E. Batcheva, The second term of the asymptotics of the monodromy map in case of two even edges of Newton diagram, Electron. J. Qual. Th. Diff. Eqs. 19 (2000), 1-15.

[30] R. Moussu, Symétrie et forme normale des centres et foyers dégénérés, Ergodic Theory and Dynam. Systems 2 (1982), 241-251.

[31] R. Moussu, Une démonstration d'un théorème de Lyapunov-Poincaré, Astérisque 98-99 (1982), 216-223.

[32] H. Poincaré, Sur l'intégration des équations différentielles du premier ordre et du premier degré $I$ and II, Rendiconti del circolo matematico di Palermo $\mathbf{5}$ (1891), 161-191; 11 (1897), 193-239.

[33] E. Strózyna \& H. ŻoŁA̧DEK, The analytic and normal form for the nilpotent singularity, J. Differential Equations 179 (2002), 479-537.

[34] H. ŻOŁA̧DEK, The monodromy group. Mathematics Institute of the Polish Academy of Sciences. Mathematical Monographs (New Series), 67. Birkhuser Verlag, Basel, 2006.

[35] H. Żo€A̧DeK \& J. Llibre, The Poincaré center problem, J. Dyn. Control Syst. 14 (2008), no. 4, 505-535.

Departament de Matemàtica, Universitat de Lleida, Av. Jaume II, 69, 25001 Lleida, Spain

E-mail address: gine@matematica.udl.cat

Departament de Matemàtiques, Universitat Autònoma de Barcelona, 08193 Bellaterra, Barcelona, Spain

E-mail address: jllibre@mat.uab.cat 